\title{
Periareolar incision for the management of benign breast tumors
}

\author{
XIANGNAN KONG $^{1 *}$, XI CHEN $^{1 *}$, LIYU JIANG $^{1}$, TINGTING MA ${ }^{1}$, BAOSAN HAN ${ }^{2,3}$ and QIFENG YANG ${ }^{1,4}$ \\ ${ }^{1}$ Department of Breast Surgery, Qilu Hospital, Shandong University, Jinan, Shandong 250012; \\ ${ }^{2}$ Department of General Surgery, School of Medicine, Shanghai Jiao Tong University; ${ }^{3}$ Laboratory of General Surgery, \\ School of Medicine, Xinhua Hospital Affiliated to Shanghai Jiao Tong University, Shanghai 200092; \\ ${ }^{4}$ Pathology Tissue Bank, Qilu Hospital, Shandong University, Jinan, Shandong 250012, P.R. China
}

Received December 3, 2015; Accepted June 27, 2016

DOI: $10.3892 / \mathrm{ol} .2016 .5117$

\begin{abstract}
Benign breast tumors (BBTs) are common in women. The traditional surgical resection method for the various types of BBT leaves obvious scars and affects the appearance of the breast. The present study introduces the experience of a single institution in the treatment of BBT by periareolar incision. The clinical data of 153 patients (182 breasts) with BBT who had undergone a resection via a periareolar incision between January 2010 and December 2012 in Qilu Hospital, Shandong University (Jinan, Shandong, China), was retrospectively analyzed. All incisions were primary healing. Of the 153 patients, $1(0.7 \%)$ developed a hematoma and $2(1.3 \%)$ developed slight nipple ischemia. No infections or other complications were observed. During 1 month to 3 years of follow-up, the cosmetic effects were assessed. Periareolar incision is not only suitable for all types of breast surgery for benign tumor resection, but also has the advantage of a hidden incision, a small scar, no ischemic necrosis of the nipple areola, high patient satisfaction and good post-operative cosmetic effect. The technique is therefore a good surgical incision choice that is worthy of note.
\end{abstract}

\section{Introduction}

Breast disease seriously affects the physical and mental health of women. In recent years, the incidence rates of benign breast tumors (BBTs) and malignant tumors have been rising.

Correspondence to: Dr Baosan Han, Department of General Surgery, School of Medicine, Shanghai Jiao Tong University, 1665 Kongjiang Road, Shanghai 200092, P.R. China

E-mail: hanbaosan@126.com

Dr Qifeng Yang, Department of Breast Surgery, Qilu Hospital, Shandong University, 107 Wenhua Xi Road, Jinan, Shandong 250012, P.R. China

E-mail: qifengy_sdu@163.com

${ }^{*}$ Contributed equally

Key words: benign breast tumor, fibroadenoma, intraductal papilloma, periareolar incision, cosmetology
BBTs are much more common than malignant breast cancer, accounting for $>90 \%$ of referrals to secondary care $(1,2)$. BBTs include fibroadenoma, intraductal papilloma and lipomyoma, among others (3). There is a possibility for malignant transformation in BBTs. Surgical resection is the main method for the treatment of the tumors (4).

The traditional method of BBT resection used at our hospital is a radial or arc incision along the dermatoglyph on the skin above the pathological changes of the breast, in order to minimize damage to the mammary ducts. However, obvious post-operative scarring seriously affects the appearance of the breast. If there are multiple lesions, there will be more scars left after removal of the tumors and the bilateral breasts will be asymmetrical. This can cause the patient much psychological pressure and affect their quality of life.

With the continuous development of the social economy and the gradual improvement of living standards, demands for breast appearance are higher. Surgery should not only cure the breast disease, but it should also avoid damage to the functions and appearance of the breasts. Therefore the choice of method for BBT resection should aim to minimize obvious scarring and maintain the overall appearance of the breasts.

In our experience at Qilu Hospital, Shandong University (Jinan, China), the periareolar incision should be considered the first choice for BBT. This allows good treatment of the BBT and minimal local periareolar scarring. When minimal external scarring and changes to the overall appearance of the breast are requested by the patients, a periareolar incision is a good solution. The present study reports the results of the use of this technique by a single institution in a retrospective analysis of 153 BBT patients.

\section{Materials and methods}

Patients. A total of 153 patients (182 breasts) operated upon using a periareolar incision between January 2010 and December 2012 were enrolled in this retrospective study. Patient information and written consent forms were obtained from the Pathology Tissue Bank of Qilu Hospital. The present study was approved by Medical Ethics Committee of of Qilu Hospital. Patient ages ranged between 14 and 62 years old. The clinical characteristics of the patients are listed in Table I. Of all the patients with breast masses, 92 had a single mass and 70 had multiple masses. The remaining breasts were operated 
A

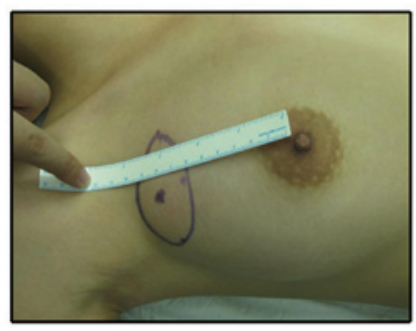

D

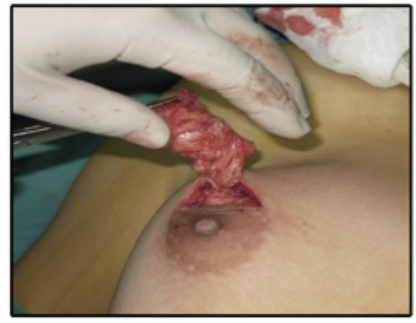

B

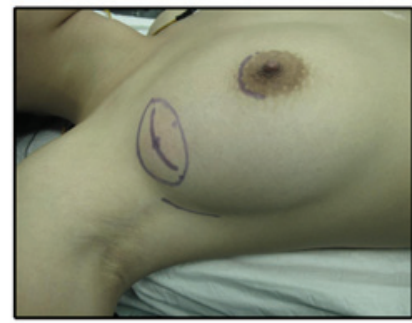

$\mathbf{E}$

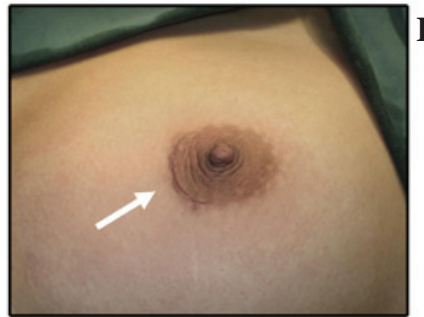

C
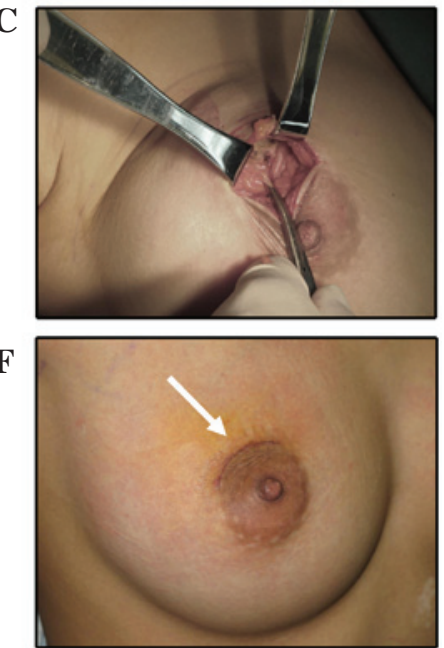

Figure 1. Surgical procedures. (A) The surface projection of the mass was marked on the skin and the distance from the mass to the areola was measured. (B) For this tumor, three types of incision could be chosen, but the periareolar incision was selected. (C) The incision was opened and the mass was pulled with forceps towards the incision. (D) The mass, together with part of its surrounding normal glands and adipose tissue, were completely removed. (E) The incision was sutured and glued using medical biological glue. (F) The results 3 days after the surgery. White arrows show the incisions.

on due to nipple discharge. The masses of 40 breasts were located under or around the areola, while other masses located in each quadrant of the breast. The longest distance from the mass to the edge of the areola was $7.5 \mathrm{~cm}$. The largest mass was $5 \times 3 \mathrm{~cm}$ and the smallest was $0.5 \times 0.5 \mathrm{~cm}$. Pre-operative examination (ultrasonography or mammography) was performed to exclude the malignant tumors. Table II shows the association between the surgical method and the type of BBT.

The follow-up time ranged between 1 month and 3 years, and the results were evaluated by the person who had performed the surgery.

Surgical technique. The procedure was performed under general anesthesia and the patient was placed in a supine position. The surface projection of the mass was marked on the skin and the distance from the mass to the areola was measured. The nearest periareolar incision area to the mass was chosen. If there were multiple masses, the incision was chosen by taking into account the location of the majority of the masses or the incision on the upper outer edge of the areola was chosen to ensure the normal sensation of the nipple and areola. The length of the incision was determined based on the size of the tumor and the distance from the tumor to areola, and was no more than half of the length of the perimeter of areola.

The skin, subcutaneous tissue and superficial fascia were incised in turn until the gland surface. The incision was opened and the tissues between the subcutaneous adipose and mammary gland capsule were sharply separated until reaching the surface of the mass. If the mass was far from the areola, it was pushed or pulled with forceps towards the incision. The gland layer was radially incised and the mass, together with part of its surrounding normal glands and adipose tissue, were completely removed. Electrocoagulation was used to stop any bleeding. The surgical residual cavity was not sutured and a drainage tube was not placed. The subcutaneous adipose layer was sutured with 5-0 absorbable interrupted sutures, followed by intradermal sutures. The incision was covered using medical biological glue. After the surgery, the resection site was compressed using bandages. The aforementioned procedures involved in the mammary tumor resection are shown in Fig. 1.

For patients with multiple and relatively concentrated masses, mammary gland lobectomy, segment and quadrant resection could be performed. During the procedure, the gland layer was radially incised along the direction of the mammary ducts until the retromammary cellular space. The surgeon explored the gland layer completely using their fingers. The glands, including all the masses, were pulled below the incision. Multiple masses, together with part of the surrounding normal mammary glands, were removed completely.

For the patients who received a subcutaneous mastectomy, part of the normal mammary glands was maintained under the nipple and areola to ensure the blood supply of the nipple-areolar complex. The nipple-areola plasty was routinely performed to avoid post-operative nipple collapse.

After the surgery, the breast collapsed significantly for patients with a thin subcutaneous fatty layer and more gland tissues. For these patients, silastic gel breast prosthesis implantation could be surgically performed based on the patient's requirements.

\section{Results}

All incisions were primary healing. In the early post-operative days, swelling of the surgical area was commonly observed. Of the 153 patients $1(0.7 \%)$ developed hematoma and $2(1.3 \%)$ developed slight nipple ischemia. No infections or other complications were observed.

After 1 month to 3 years of follow-up, there was no obvious scarring, with the exception of 3 patients (2.0) with scar diathesis. In total, 2 patients underwent silastic gel breast prosthesis implantation successfully. Fig. 2 showed representative post-surgical images of 3 patients. A total of 32 breasts (17.6\%) developed areola collapse and 51 breasts $(28.0 \%)$ developed nipple paresthesia. Tables III and IV show the associations between areola 
Table I. Clinical characteristics of the patients.

\begin{tabular}{lc}
\hline Clinical data & Value \\
\hline Total patients, $\mathrm{n}$ & 153 \\
Mean age \pm standard deviation, years & $38.0 \pm 9.4$ \\
Marital and fertility status, $\mathrm{n}(\%)$ & \\
Single and nulliparous & $15(9.8)$ \\
Married and nulliparous & $13(8.5)$ \\
Married with children & $125(81.7)$ \\
Affected breasts, n (\%) & \\
Unilateral & $124(81.0)$ \\
Bilateral & $29(19.0)$ \\
Reason for treatment, $\mathrm{n}(\%)$ & $119(77.8)$ \\
Breast mass & $26(17.0)$ \\
Nipple discharge & $4(2.6)$ \\
Breast mass and nipple discharge & $4(2.6)$ \\
Other & \\
Previous breast history, n (\%) & $16(10.5)$ \\
Benign breast tumor & $2(1.3)$ \\
Contralateral breast cancer & \\
\hline
\end{tabular}

collapse, nipple paresthesia, surgical method and the type of BBT. Patient satisfaction was $98.0 \%$, with unsatisfactory results for 3 patients who had undergone subcutaneous mastectomy, but without silastic gel breast prosthesis implantation.

During the follow-up, 2 patients who underwent tumor resection and 2 patients who underwent mammary gland lobectomy experienced a lactation period after the surgery. All patients retained the ability to breastfeed.

\section{Discussion}

Plump and elastic breasts are important functionally and esthetically. Generally, conservative treatment cannot cure BBT, and surgical resection is the most effective and thorough treatment. Traditional incisions for BBT are radial or arc incisions along the dermatoglyph on the skin above the pathological changes of the breast. The surgery is easy to perform, however, its disadvantage is the obvious scarring which seriously affects the appearance of the breast, particularly in patients with multiple lesions. Nowadays, the requirements for a beautiful post-operative breast appearance are higher.

Mammary glands are located between the superficial layer and the deep layer of the superficial fascia. The superficial layer of the superficial fascia is tightly connected with the skin, and the deep layer of the superficial fascia is connected with the superficial layer of the pectoralis major fascia by loose connective tissues. Mammary tissues have good elasticity and softness (5). These anatomical structures ensure that the breast has a certain mobility and is relatively stable. The skin of the areola area is relatively thin, and has good elasticity and strength, with a wide range of resection techniques available. In the majority of cases, post-operative scarring is not obvious and can quickly heal. All these reasons make a periareolar incision possible, and surgery through periareolar incisions
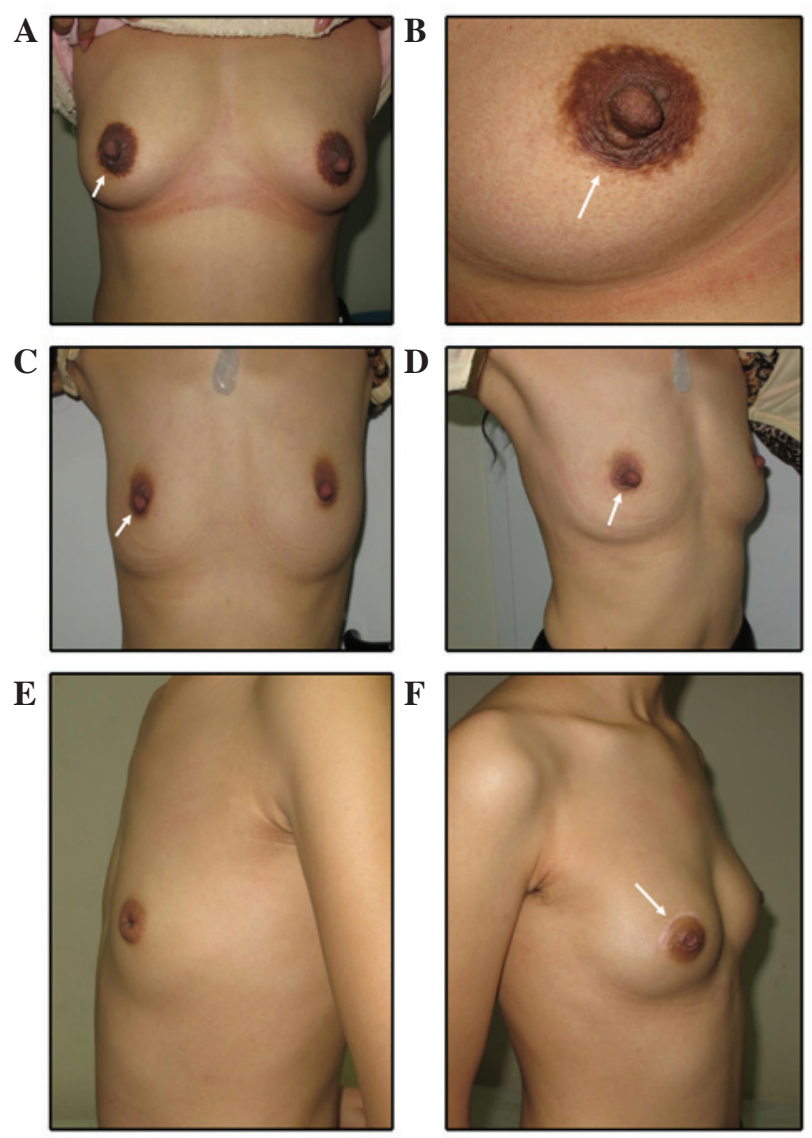

Figure 2. (A and B) Representative patient images 2 years after a mammary tumor resection. (C and D) Representative patient images 6 months after a mammary gland lobectomy. View of a patient (E) prior to and (F) 6 months after bilateral subcutaneous mastectomy with silastic gel breast prosthesis implantation. White arrows showed the incisions.

are gradually being welcomed by female patients with BBT. For the 153 patients in the present study, there were no obvious scarring, with the exception of 3 patients with scar diathesis. The patient satisfaction was high at $98 \%$. Due to its cosmetic role, periareolar incision has also been widely used in the treatment of gynecomastia (6-8).

Certain studies have previously reported that there are no differences between a periareolar incision and a traditional incision with regard to the duration of surgery or the amount of bleeding $(9,10)$. However, other studies have reported the opposite, stating that a resection through a periareolar incision requires more time $(\sim 2 \mathrm{~min})$ and results in more bleeding ( $\sim 10 \mathrm{ml}$ ) compared with the traditional surgical method (11). Although this result was supported by statistics, it has little practical significance in clinical practice. A periareolar incision causes more damage to the subcutaneous tunnel and mammary glands, thus it requires more focus on the surgical techniques, including intraoperative exposure, intraoperative bleeding and incision closure, otherwise the risk of surgical complications could increase. Of the 153 patients, only $1(0.7 \%)$ developed hematoma, while $2(1.3 \%)$ developed slight nipple ischemia. No cases were administered post-operative antibiotics and no infections occurred. It has previously been reported that a periareolar incision is not suitable when the diameter of the tumor is $>5 \mathrm{~cm}$ or when the distance between 
Table II. Association between surgical method and type of benign breast tumor.

\begin{tabular}{lcrc}
\hline Surgical method & Fibroadenoma & Intraductal papilloma & Mastoplasia \\
\hline Tumor resection, $\mathrm{n}$ & 87 & 1 & 3 \\
Gland lobectomy, segment or quadrant resection, $\mathrm{n}$ & 24 & 18 & 3 \\
Subcutaneous mastectomy, $\mathrm{n}$ & 9 & 25 & 3 \\
\hline
\end{tabular}

Table III. Association between areola collapse, nipple paresthesia and surgical method.

\begin{tabular}{lccc} 
Side effect & Tumor resection & $\begin{array}{c}\text { Gland lobectomy, segment } \\
\text { or quadrant resection }\end{array}$ & Subcutaneous mastectomy \\
\hline $\begin{array}{l}\text { Areola collapse, } \mathrm{n}(\%) \\
\text { Yes }\end{array}$ & $2(2.1)$ & $11(24.4)$ & $19(44.2)$ \\
No & $92(97.9)$ & $34(75.6)$ & $24(55.8)$ \\
Nipple paresthesia, $\mathrm{n}(\%)$ & $11(11.7)$ & & \\
$\quad$ Yes & $83(88.3)$ & $32(71.1)$ & $27(62.8)$ \\
No & & & $16(37.2)$ \\
\hline
\end{tabular}

Table IV. Association between areola collapse, nipple paresthesia and type of benign breast tumor.

\begin{tabular}{lcccr}
\hline Side effect & Fibroadenoma & Intraductal papilloma & Mastoplasia & Other \\
\hline $\begin{array}{l}\text { Areola collapse, } \mathrm{n}(\%) \\
\text { Yes }\end{array}$ & $9(7.5)$ & & & $1(9.1)$ \\
No & $111(92.5)$ & $21(47.7)$ & $10(90.9)$ & $6(85.7)$ \\
Nipple paresthesia, n (\%) & & & & $3(27.3)$ \\
Yes & $21(17.5)$ & $24(54.5)$ & $8(72.7)$ & $3(42.8)$ \\
No & $99(82.5)$ & $20(45.5)$ & & $4(57.2)$ \\
\hline
\end{tabular}

the tumor and the areola is $>3-4 \mathrm{~cm}$, since the surgery would cause significant damage to the lactiferous ducts (12). However, in the present cases, a periareolar incision was used even when the distance between the tumor and the areola was $7.5 \mathrm{~cm}$ to ensure good patient outcomes. A periareolar incision was also used in subcutaneous mastectomy with good surgical effects.

In the body, the residual cavity generally requires closure by sutures following removal of tissue, and for larger residual cavities and wound bleeding, a drainage tube should be placed to avoid complications such as subcutaneous hemorrhage and infection. However, it has been reported that for patients with more mammary tissues, the suture of the residual cavity is more difficult. Thus, if the residual cavity was forced closed by sutures, it would cause appearance changes of varying degrees (13). In the 153 patients of the present study, electrocoagulation was chosen to stop any bleeding instead of suturing the residual cavity. This method did not cause appearance changes of the breast, and following the surgery, discharge could fill the residual cavity and maintain the appearance of the breast. Within 3 months of the surgery, the residual cavity narrowed gradually, probably as it was filled by fiber granulation tissues, and the discharge was absorbed. Additionally, drainage tubes were not placed in any of the 153 patients.
Instead, compression was applied using bandages. This reduced the exudation of the surgical area and contributed to the recovery of the breast. Particular attention was paid to avoid compression of the incision, nipple and areola, so as to prevent necrosis of the skin edge and incision flap, and ischemic necrosis of the nipple and areola.

The only drawback of periareolar incision with regard to appearance is the possibility of flatness or collapse of the nipple-areola area. Reasons for this include i) the characteristics of the breast, such as more mammary glands and a thin fatty layer; ii) surgical factors, such as too much resection of the subcutaneous mammary tissues, particularly tissues under the nipple-areola area; and iii) breast mass factors, such as a large mass occupying the whole breast or a tumor located in the deep areola. Of the 182 breasts, 32 developed nipple collapse. Of these, 21 (65.6\%) initially exhibited intraductal papilloma (IDP). The treatment of IDP often requires a larger extent of resection and greater resection of tissues under the areolar area. This may be the reason why $17.6 \%(n=32)$ of breasts developed nipple collapse.

Additionally, 51 breasts $(28.0 \%)$ developed nipple paresthesia. It can be concluded that the majority of patients who had developed nipple paresthesia initially presented with IDP 
and underwent surgery to a greater extent. A previous study reported that the incidence of nipple paresthesia for periareolar incision was higher compared with that found for traditional incision (11). We hypothesize that when the extent of surgery is larger it is more likely to damage the nerve endings. The incision could also be overstretched in the surgery causing nerve injury. A study also reported that the differences in the times of sensory recovery for different locations and lengths showed no statistical significances. Therefore, the location and cut length by periareolar incision in treatment of BBT could be chosen according to the location and size of the tumor, which did not affect the time of the sensory recovery of the skin (14). During the follow-up in the present study, it was found that patients with nipple paresthesia gradually recovered over time. No irreversible damage occurred.

Subcutaneous mastectomy has good treatment effects for certain types of BBT. However, it seriously affects the appearance of the breast and brings a great psychological burden to patients. Therefore, for patients with higher cosmetic requirements, silastic gel breast prosthesis implantations can be performed at the same time as the subcutaneous mastectomy. Of the 153 patients, only 2 underwent silastic gel breast prosthesis implantation. Subsequent to the surgery, the bilateral breasts were symmetric with a good appearance, and the patient satisfaction was high.

In conclusion, a periareolar incision brings hope to female patients with BBT; it enables the disease to be cured while maintaining the appearance of the breast. A quick recovery, hidden incision, small scar and other advantages mean that the periareolar incision should be used a main surgical technique for BBT. However, periareolar incision requires higher technical requirements from the surgeons and may cause areola collapse. From the point of the appearance of the breast, we advise patients with high cosmetic requirements to receive silastic gel breast prosthesis implantations in order to maintain a good breast shape.

\section{Acknowledgements}

This study was supported by the National Natural Science Foundation of China (grant nos. 81172529 and 81272903) and the Shandong Science and Technology Development Plan (grant no. 2013GRC31801).

\section{References}

1. Chen W, Zheng R, Zhang S, Zhao P, Li G, Wu L and He J: The incidences and mortalities of major cancers in China, 2009. Chin J Cancer 32: 106-112, 2013

2. Onstad M and Stuckey A: Benign breast disorders. Obstet Gynecol Clin North Am 40: 459-473, 2013.

3. Laufer M and Goldstein D: The breast: Examination and lesions. In Pediatric and Adolescent Gynecology. 5th edition. Sydor A (ed). Lippincott Williams and Wilkins, Philidelphia, pp729-759, 2005.

4. Dixon JM, Dobie V, Lamb J, Walsh JS and Chetty U: Assessment of the acceptability of conservative management of fibroadenoma of the breast. Br J Surg 83: 264-265, 1996.

5. Hamdi M, Würinger E, Schlenz I and Kuzbari R: Anatomy of the breast: A clinical application. In: Vertical Scar Mammaplasty. Springer, pp1-8, 2005

6. Lapid O, Klinkenbijl JH, Oomen MW and van Wingerden JJ: Gynaecomastia surgery in the Netherlands: What, why, who, where. J Plast Reconstr Aesthet Surg 67: 702-706, 2014.

7. Lee JH, Kim IK, Kim TG and Kim YH: Surgical correction of gynecomastia with minimal scarring. Aesthetic Plast Surg 36: 1302-1306, 2012.

8. Cannistra C, Piedimonte A and Albonico F: Surgical treatment of gynecomastia with severe ptosis: Periareolar incision and dermal double areolar pedicle technique. Aesthetic Plast Surg 33: 834-837, 2009.

9. Chen XY: Clinical observation on surgery through a minimal incision around areola mammae in treatment of benign tumor of mammary glands. Hebei Yixue 20: 777-780, 2014 (In Chinese).

10. Hu N: The clinical effects of different resection type of breast Fibroids. Zhongguo YIyao Daokan 15: 1135-1137, 2013 (In Chinese).

11. Liu XF, Zhang JX, Zhou Q, Chen F, Shao ZM and Lu C: A clinical study on the resection of breast fibroadenoma using two types of incision. Scand J Surg 100: 147-152, 2011.

12. Zhao XY: Application value of areola incision at the edge of the benign breast tumor resection. Zhongguo Shiyong Yiyao 5: 57-58, 2010 (In Chinese).

13. Cao MZ: An improvement for dead-space management in partial mastectomy. Qingdao Daxue Yixueyuan Xuebao 39: 235-236, 2003 (In Chinese).

14. Kong LW, Ma XJ, Liu YH, Wang J and Han JQ: Influence of the circumareolar incision exsection in treatment of benign breast tumour on the nipple-areola complex sensibility. Jiefangjun Yiyao Zazhi 26: 84-86, 2014 (In Chinese). 